BMJ Open

Diabetes

Research

\& Care

\title{
Transfer in care and diabetes distress in young adults with type 1 diabetes mellitus
}

\author{
Jane Sattoe (1) , ${ }^{1,2}$ Mariëlle Peeters, ${ }^{1,2}$ Madelon Bronner, ${ }^{2}$ AnneLoes van Staa ${ }^{1,2}$
}

To cite: Sattoe J, Peeters M, Bronner $\mathrm{M}$, et al. Transfer in care and diabetes distress in young adults with type 1 diabetes

mellitus. BMJ Open Diab Res Care 2021;9:e002603. doi:10.1136/

bmjdrc-2021-002603

Received 14 September 2021 Accepted 23 November 2021
Check for updates

(C) Author(s) (or their employer(s)) 2021. Re-use permitted under CC BY-NC. No commercial re-use. See rights and permissions. Published by BMJ.

${ }^{1}$ Erasmus School of Health Policy \& Management, Erasmus University Rotterdam, Rotterdam, The Netherlands ${ }^{2}$ Research Centre Innovations in Care, Rotterdam University of Applied Sciences, Rotterdam, The Netherlands

Correspondence to Dr Jane Sattoe; sattoe@eshpm.eur.nl

\section{ABSTRACT}

Introduction Diabetes distress (DD) is a serious problem in many people with diabetes and is associated with unfavorable clinical and psychosocial outcomes in children and adults. Little is known about DD in young adults (YAs) with type 1 diabetes mellitus (T1DM) who transferred to adult care. This study aimed to explore the differences between YAs with/without DD regarding transfer experiences, self-management and health-related quality of life (HRQOL).

Research design and methods Cross-sectional online questionnaire completed by YAs with T1DM after transfer. DD was measured with the short-form Problem Areas in Diabetes scale. Descriptive analyses were followed by t-tests and $\chi^{2}$ tests to explore differences between the groups with/without DD. Effect sizes were calculated. Results $0 f 164$ respondents with mean age $22.7( \pm 1.56)$ years, $60.7 \%$ was female. The total sample scored low on DD (6.52 \pm 4.67 ; range: $0-17)$, but $57(34.8 \%)$ had a score $\geq 8$, indicating DD. YAs with DD felt less ready to transfer to adult care than those without DD and scored lower on alliance between pediatric and adult care and reception in adult care. They also reported poorer selfmanagement skills and lower HRQOL in all domains of functioning.

Conclusions More than one-third YAs experienced DD after transfer; this was associated with less favorable transition, self-management and psychosocial outcomes. Transfer in care seems to be a source of DD. Systematic screening on DD and attention for YAs' worries is recommended in both pediatric and adult care.

\section{INTRODUCTION}

Emerging adulthood is a critical period with increased risk of acute and even chronic health complications, psychological problems and psychosocial issues for young adults with type 1 diabetes mellitus (T1DM). ${ }^{1}$ Young people growing up with T1DM go through various transitions while transitioning from childhood to adulthood. Next to the developmental milestones they are expected to achieve, they are required to transfer to adult care and take up self-management of their chronic condition, which can be challenging. ${ }^{2}{ }^{3}$ Young adults are underrepresented in research on living with T1DM, although they have unique views, challenges

\section{Significance of this study}

What is already known about this subject?

- The few studies that explored diabetes distress in young adults with type 1 diabetes mellitus (T1DM) showed that diabetes distress is significantly more present in the young adult group compared with other age groups and that more diabetes distress in young adults with T1DM is associated with impaired health outcomes and less frequent blood monitoring by young adults.

\section{What are the new findings?}

- More than one-third of the young adults with T1DM experienced diabetes distress after transfer to adult care.

- Having diabetes distress was associated with less favorable transition experiences, self-management outcomes and psychosocial outcomes.

- Transfer in care may be a source of diabetes distress in young adults.

How might these results change the focus of research or clinical practice?

- Systematic screening on diabetes distress and attention for young adults' worries is recommended. Building positive relationships and adopting motivating communication styles may help clinicians to reduce diabetes distress in this population.

and needs. ${ }^{3} 4$ The regular developmental tasks of young adults in emerging adulthood may influence and be influenced by T1DM. ${ }^{2}$ Diabetes management, for instance, is often a lower priority for young adults with T1DM during important life transitions, ${ }^{5}$ and glycaemic control of this group is less optimal compared with other age groups. ${ }^{1}$

Studies repeatedly show that living with diabetes comes with a profound emotional burden. ${ }^{6}$ Consequently, young people with T1DM are at significant risk for psychological comorbidity: high prevalence of depressive symptoms, anxiety and eating disorders have been reported in this group. ${ }^{7-10}$ Apart from these clinically defined psychological diagnoses, diabetes distress is more and 
more recognized as an important indicator for mental health. ${ }^{11} 12$ Diabetes distress is defined as 'the negative emotional or affective experience resulting from the challenge of living with the demands of diabetes.... ${ }^{13}$ It is associated with unfavorable clinical and psychosocial outcomes and lower levels of self-management. ${ }^{12}$ Different studies found the number of people experiencing diabetes distress to be higher than the number of people experiencing clinically established psychological disorders such as depression ${ }^{13} 14$ Therefore, diabetes distress can be a more useful indicator to gain insight into well-being and needs of (young) people with diabetes, and to prevent further deterioration of mental health in this group. This is why international diabetes guidelines recommend regular screening on diabetes distress in clinical practice. ${ }^{12}$

Diabetes distress has been topic of research for at least 25 years now, ${ }^{13}$ but relatively little research has specifically addressed young adults. ${ }^{615}$ The few studies that explored this area showed that diabetes distress is significantly more present in the young adult group compared with other age groups ${ }^{56}$ and that more diabetes distress in young adults with T1DM is associated with impaired health outcomes (eg, higher hemoglobin A1c (HbA1c) levels) ${ }^{1516}$ and less frequent blood monitoring by young adults. ${ }^{17}$ Garvey and colleagues found that feeling prepared for transition to adult care was associated with lower diabetes distress in young adults with T1DM. ${ }^{18}$ However, this is the only study that addressed transition and diabetes distress. Since transition is an important phase to support the development of self-management skills and to prepare young people for adult life while maintaining good quality of life, it is useful to further explore the differences between young people with and without diabetes distress regarding transfer experiences, self-management and health-related quality of life. Such insights could help to tailor self-management support to the needs of young adults. This study aimed to explore these differences in a sample of Dutch young adults (aged 18-25 years) with T1DM.

\section{RESEARCH DESIGN AND METHODS \\ Participants and setting}

This study used data from a larger evaluation study of transitional care for young adults with T1DM in the Netherlands. ${ }^{19} 20$ The study involved 12 hospitals and was conducted between April 2016 and October 2018; its study protocol has already been published, ${ }^{21}$ as is the evaluation study itself. ${ }^{19}$ In the current study, results from an online questionnaire on diabetes distress, healthrelated quality of life, self-management and transfer experiences were explored. Participants were young adults (aged 18-25 years) with a confirmed diagnosis of T1DM who had made the transfer to adult services in 2012-2014, had no cognitive impairment and were able to speak and read Dutch. In our evaluation study, we found that the care facilities could be divided into two groups: HI-ATT (with high attention for transitional care and thus better alliance between pediatric and adult care settings) and LO-ATT (the opposite). We established that there were hardly any significant differences between the two groups related to outcomes in young adults, such as health-related quality of life and self-management. Extra analysis (not published) also showed that there were no differences in diabetes distress. That is why in this study, we did not differentiate between teams with more or less transitional care facilities.

\section{Data collection}

The participating hospitals invited potential respondents by email in October 2017. They were asked to fill out an online questionnaire on diabetes distress, health-related quality of life, self-management and transfer experiences. Most invited people had transferred to adult services in the period 2012-2014, but five hospitals also invited young adults who had transferred in 2015-2016. Reminders were sent after 2 and 4 weeks. Every third respondent received $\mathrm{a} € 20$ gift voucher. Those who eventually participated, consented with the use of their data and linking these to data from their electronic charts for this study.

\section{Measures}

Background characteristics

The following sociodemographic characteristics were addressed in the questionnaire: age (years); gender (male/female); highest completed educational level at time of the study (low (ie, junior vocational or secondary general low) and medium (ie, secondary general high or senior vocational) versus high (higher educational institutions)); employment status (paid or volunteer job, yes vs no) and living status (with parents vs independent). Time since transfer (first consultation in adult care) had been collected in the overarching evaluation study from participants' medical records.

\section{Diabetes distress}

Diabetes distress was measured with the Dutch version of the validated 5-item Problem Areas in Diabetes (PAID-5) scale. ${ }^{2223}$ Items are scored on a 5-point Likert scale: $0=$ not a problem, $1=$ minor problem, $2=$ moderate problem, $3=$ somewhat serious problem, $4=$ serious problem. The theoretical range is $0-20$; higher scores indicate more stress. Cronbach's $\alpha$ in the current study was 0.89 . For the categorization of groups into 'with diabetes distress' and 'without diabetes distress', a score of eight points or higher on the sum score was classified as having diabetes distress. $^{22}$

\section{Transfer experiences}

Transfer experiences were measured using the validated 20-item On Your Own Feet-Transfer Experiences Scale. ${ }^{24}$ Items are scored on a 5-point Likert scale: 1=strongly disagree, $2=$ disagree, $3=$ do not agree/do not disagree, $4=$ agree, $5=$ strongly agree. Higher scores indicate more positive transfer experiences. Cronbach's $\alpha$ for the total 
scale was 0.91 in the current study. Subscales included: reception in adult care (four items, $\alpha=0.88$, alliance between pediatric and adult care (five items, $\alpha=0.87$ ), preparation for the transfer (three items, $\alpha=0.70$ ), readiness to transfer (six items, $\alpha=0.81$ ) and youth involvement (two items, $\alpha=0.60$ ).

\section{Self-management}

Self-management skills were measured with the validated 12-item Partners in Health scale. ${ }^{25}$ Items are scored on a 9-point Likert scale, where a higher score indicates better self-management skills. Cronbach's $\alpha$ in the current study was 0.74 .

\section{Health-related Quality of Life}

Health-related Quality of Life (HRQoL) was measured using the Dutch version of the validated 23-item Pediatric Quality of Life Inventory for Young Adults. ${ }^{26}$ Items are scored on a 5 -point Likert scale: $0=$ never, $1=$ almost never, $2=$ sometimes, $3=$ often, $4=$ almost always. Each answer is reversely scored and rescaled to a $0-100$ scale $(0=100$, $1=75,2=50,3=25$, and $4=0$ ). Higher scores indicate better HRQoL. Cronbach's $\alpha$ for the total scale was 0.94 in the current study. Subscales included: physical health (eight items, $\alpha=0.88$, emotional functioning (five items, $\alpha=0.86$ ), social functioning (five items, $\alpha=0.80$ ) and school/work functioning (five items, $\alpha=0.80$ ).

\section{Data analysis}

Descriptive analyses were performed to describe the study sample's background characteristics. Differences on background characteristics between non-responders and responders were calculated using t-tests and Pearson $\chi^{2}$ tests. The study sample was divided into two groups on the basis the PAID-5 scale score: (1) with diabetes distress (score 28 ) and (2) without diabetes distress (score $<8$ ). Differences between these two groups were explored with t-tests and Pearson $\chi^{2}$ tests and effect sizes (Cohen's $d$ ) were calculated; descriptive analyses (frequencies) were used to explore diabetes distress within these groups (at item level). Missing data were excluded listwise.

\section{RESULTS}

\section{Response and background characteristics}

Four hundred and thirty-five young adults with T1DM were invited to participate in the study. Of these, 177 $(40.7 \%)$ filled out the questionnaire and $164(37.7 \%)$ provided information about diabetes distress. Nonresponders did not differ from the responders in age (mean \pm SD in years: $23.6 \pm 1.40$ vs $23.9 \pm 1.49$; $p=0.158$ ), but they were more often men ( $64.0 \%$ vs $39.3 \%$; $\mathrm{p}<0.001)$. A description of the study sample is given in table 1 .

\section{Diabetes distress}

The total study sample $(\mathrm{n}=164)$ scored low on problem areas in diabetes $(6.52 \pm 4.67$, range: $0-17)$ but 57 of them $(34.8 \%)$ had a score $\geq 8$, indicating diabetes distress. These young adults with diabetes distress had a mean

\begin{tabular}{|c|c|}
\hline Characteristic & $\begin{array}{l}\text { Mean }( \pm S D) \text { or } \\
\text { Frequency }(\%)\end{array}$ \\
\hline Age (years) (range 19-28 years) & $22.7( \pm 1.56)$ \\
\hline Time since transfer ${ }^{\star}(3-6$ years) & $4.7( \pm 1.13)$ \\
\hline \multicolumn{2}{|l|}{ Gendert } \\
\hline Female & 99 (60.7\%) \\
\hline Male & $64(39.3 \%)$ \\
\hline \multicolumn{2}{|l|}{ Educational level $\dagger$} \\
\hline Low/middle & $125(76.7 \%)$ \\
\hline High & $38(23.3 \%)$ \\
\hline \multicolumn{2}{|l|}{ Paid or volunteer job† } \\
\hline Yes & $137(84.0 \%)$ \\
\hline No & $26(16.0 \%)$ \\
\hline \multicolumn{2}{|l|}{ Living† } \\
\hline With parents & $99(60.7 \%)$ \\
\hline Independent (on own) & 64 (39.3\%) \\
\hline
\end{tabular}

${ }^{*} \mathrm{n}=112$.

$\dagger \mathrm{n}=163$.

distress score of $11.88( \pm 2.99)$ vs $3.66( \pm 2.27)$ in those without diabetes distress $(\mathrm{p}<0.001)$. 'Worrying about the future and the possibility of serious complications' was most often mentioned as a serious problem by those with diabetes distress $(67 \%$; figure 1$)$. Those without diabetes distress also worried about this, but only $5 \%$ considered it a serious problem. 'Feeling that diabetes is taking up too much of your mental and physical energy every day' was relatively often mentioned as a serious problem as well by $58 \%$ of those with diabetes distress. Comparisons of those with and those without diabetes distress on the PAID-5 showed substantial and significant differences between both groups (figure 1).

\section{Differences on background characteristics and outcomes}

Interestingly, there were hardly any differences in background characteristics between young adults with and without diabetes distress (table 2). However, the latter were significantly younger $(\mathrm{p}=0.038)$.

As for transfer experiences, young adults with diabetes distress overall had less positive experiences $(\mathrm{p}=0.002)$. More specifically, they rated the reception in adult care $(\mathrm{p}=0.013)$ and the alliance between pediatric and adult care $(\mathrm{p}=0.029)$ lower and felt less ready to transfer to adult care $(\mathrm{p}<0.001)$. They also reported worse outcomes on self-management $(\mathrm{p}<0.001)$ and overall HRQoL $(\mathrm{p}<0.001)$ compared with young adults without diabetes distress. HRQoL of those with diabetes distress was significantly lower on all domains, that is, physical functioning, emotional functioning, social functioning and school/ work functioning. Effect sizes differed from medium to large for all outcome measures. Large effect sizes were 


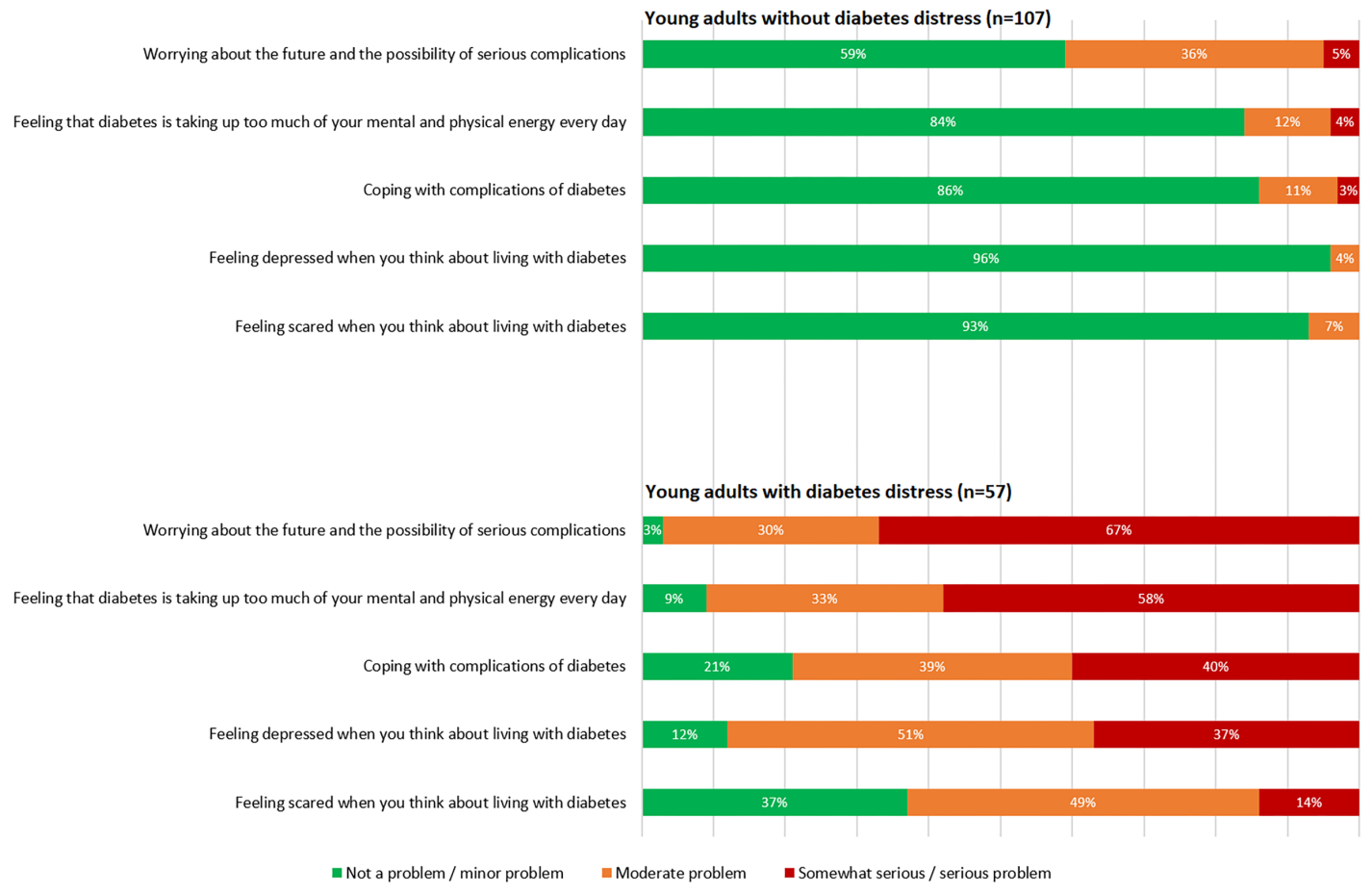

Figure 1 PAID-5 scores in young adults with and without diabetes distress. PAID, Problem Areas in Diabetes scale.

particularly found for emotional, social and school/work functioning (table 2).

\section{DISCUSSION}

\section{General discussion}

This study found substantial differences in transfer experiences, self-management and HRQoL between young adults with T1DM with and without diabetes distress. Those without diabetes distress reported better transfer experiences and better self-management skills and higher HRQoL compared with those with diabetes distress. Young adults with diabetes distress were slightly older; no other differences in background characteristics were found.

Almost $35 \%$ of the participants reported diabetes distress. The estimated prevalence of diabetes distress in other studies is $28 \%$ in adolescents and emerging adults with $\mathrm{T}^{\mathrm{DDM}}{ }^{27}$ and $32 \%$ in young adults with $\mathrm{T} 1 \mathrm{DM},{ }^{28}$ which is comparable to the present study. The outcome that those with diabetes distress were older than those without is congruent with the finding of Lašaite and colleagues (2016) that emerging young adults more often experienced psychological distress compared with adolescents. ${ }^{27} \mathrm{~A}$ study in over 19-year-olds with T1DM found that younger adults more often experienced diabetes distress than older adults. ${ }^{29}$ Probably, the developmental challenges of young adulthood add to the distress experienced from T1DM itself, which warrants specific attention from healthcare professionals. Wentzell and colleagues indeed concluded in their integrative review that diabetes distress 'is embedded within the developmental challenges specific to living with T1DM during this phase [i.e. emerging adulthood]'. ${ }^{6}$ In this light, support of parents is also an important topic. Our study revealed that less young adults who still lived with their parents had diabetes distress compared with those who lived independently. Shaw and colleagues (2021) recently emphasized the (often overlooked) role of parents in promoting young adults' well-being. ${ }^{30}$

Worrying about the future and the possibility of serious complications were most often reported as (serious) problems by people with T1DM, in our study and in previous studies as well. ${ }^{13}$ In general, distress in people with T1DM seems to be related to emotions (worries, feelings and so on) rather than difficulties in practically managing the condition and its treatment. ${ }^{31}$ However, insights into specific sources of distress in young adults with T1DM is lacking. In this study, the feeling that diabetes is taking up too much mental and physical energy every day, is the second most often mentioned problem, both by respondents with and those without diabetes distress. This problem was also highlighted in our study on HRQoL in these young adults, of whom one quarter reported fatigue due to T1DM. ${ }^{32}$ Ways to counteract the feeling that diabetes management takes up too much mental and physical energy would benefit young adults with T1DM. Nevertheless, Barry-Menkhaus and colleagues recently emphasized that much work is still needed to find pragmatic and efficient ways to improve self-management of young adults with T1DM. ${ }^{33}$ Another systematic review also highlights the need to develop effective interventions for this group. ${ }^{34}$

As for the differences in outcomes between young adults with and without diabetes distress, the latter 
Table 2 Differences between young adults with and without diabetes distress (mean $( \pm$ SD) or frequency (\%))

\begin{tabular}{|c|c|c|c|c|}
\hline & $\begin{array}{l}\text { Young adults with diabetes } \\
\text { distress }(n=57)\end{array}$ & $\begin{array}{l}\text { Young adults without diabetes } \\
\text { distress }(n=107)\end{array}$ & Effect size & $P$ value \\
\hline \multicolumn{5}{|l|}{ Background characteristics } \\
\hline Age & $23.00( \pm 1.67)$ & $22.47( \pm 1.48)$ & 0.33 & 0.038 \\
\hline Time since transfer (3-6 years) ${ }^{*}$ & $4.44( \pm 1.25)$ & $4.82( \pm 1.03)$ & 0.33 & 0.087 \\
\hline \multicolumn{5}{|l|}{ Gender† } \\
\hline Female & $39(68.4 \%)$ & $60(56.6 \%)$ & - & 0.141 \\
\hline Male & $18(31.6 \%)$ & $46(43.4 \%)$ & & \\
\hline \multicolumn{5}{|l|}{ Educational levelł } \\
\hline Low/middle & $44(78.6 \%)$ & $81(75.7 \%)$ & - & 0.681 \\
\hline High & $12(21.4 \%)$ & $26(24.3 \%)$ & & \\
\hline \multicolumn{5}{|l|}{ Paid or volunteer job $\ddagger$} \\
\hline Yes & $46(82.1 \%)$ & $91(85.0 \%)$ & - & 0.318 \\
\hline No & $10(17.9 \%)$ & $16(15.0 \%)$ & & \\
\hline \multicolumn{5}{|l|}{ Living } \\
\hline With parents & $30(53.6 \%)$ & $69(64.5 \%)$ & - & 0.188 \\
\hline On own & $26(46.4 \%)$ & $38(35.5 \%)$ & & \\
\hline \multicolumn{5}{|l|}{ Transfer experiences (OYOF-TES) } \\
\hline Reception in adult care & $3.72( \pm 0.993)$ & $4.04( \pm 0.675)$ & 0.38 & 0.013 \\
\hline $\begin{array}{l}\text { Alliance between pediatric and } \\
\text { adult care }\end{array}$ & $2.92( \pm 0.899)$ & $3.23( \pm 0.857)$ & 0.35 & 0.029 \\
\hline Preparation for the transfer & $2.88( \pm 0.925)$ & $3.02( \pm 0.931)$ & 0.15 & 0.323 \\
\hline Readiness to transfer & $3.76( \pm 0.710)$ & $4.19( \pm 0.576)$ & 0.67 & $<0.001$ \\
\hline Youth involvement & $3.12( \pm 1.02)$ & $3.39( \pm 0.955)$ & 0.27 & 0.101 \\
\hline Total score & $66.88( \pm 13.94)$ & $73.35( \pm 11.25)$ & 0.51 & 0.002 \\
\hline \multicolumn{5}{|l|}{ Self-management } \\
\hline $\mathrm{PIH}$ & $76.09( \pm 8.81)$ & $82.22( \pm 7.91)$ & 0.73 & $<0.001$ \\
\hline \multicolumn{5}{|l|}{ HRQOL (PedsQL-YA) } \\
\hline Physical functioning & $79.11( \pm 15.30)$ & $87.21( \pm 15.51)$ & 0.53 & 0.002 \\
\hline Emotional functioning & $52.81( \pm 17.70)$ & $80.28( \pm 17.43)$ & 1.56 & $<0.001$ \\
\hline Social functioning & $78.51( \pm 16.26)$ & $90.23( \pm 12.69)$ & 0.80 & $<0.001$ \\
\hline School/work functioning $†$ & $61.40( \pm 19.43)$ & $75.99( \pm 16.70)$ & 0.81 & $<0.001$ \\
\hline Total score & $69.41( \pm 14.35)$ & $83.96( \pm 13.16)$ & 1.06 & $<0.001$ \\
\hline
\end{tabular}

${ }^{*} n=112$ : Young adults with diabetes distress $n=41$, without diabetes distress $n=71$.

$\dagger n=163$ : Young adults with diabetes distress $n=57$, without diabetes distress $n=106$.

$\ddagger n=163$ : Young adults with diabetes distress $n=56$, without diabetes distress $n=107$.

HRQOL, health-related quality of life; OYOF-TES, On Your Own Feet Transfer Experiences Scale; PedsQL-YA, Pediatric Quality of Life Inventory-Young Adult; PIH, Partners in Health.

reported more positive outcomes on all measures. They had better transfer experiences, more self-management skills and higher HRQoL. It is important to note that it is still not clear whether less positive transfer experiences add to diabetes distress or - vice versa - whether diabetes distress makes young adults more negative towards past experiences, including transfer. The only previous study that explored transition and diabetes distress showed that feeling prepared for the transfer to adult care was associated with lower diabetes distress in young adults. ${ }^{18}$ However, our results suggest that rather than preparation for transfer, reception in adult care, alliance between pediatric and adult care and readiness to transfer seem to be important for young people with T1DM. This suggests that the transfer in care adds to stress experiences by young adults. More research is needed to clarify the sources of diabetes distress in young adults with T1DM.

The finding that young adults without diabetes distress score significantly better on self-management than do those with diabetes distress is not surprising. Selfmanagement encompasses three tasks: medical management, role management and emotional management. ${ }^{35}$ 
An association between lower self-care skills (medical management) and diabetes distress is consistently found in other age groups. ${ }^{13}$ Less is known about the effect of diabetes distress on social participation (ie, the degree to which someone takes up roles in society in different areas like school, work, relationships and so on), but we do know that young adults with social participation rates comparable to those of their healthy peers, generally report lower HRQoL. ${ }^{36}$ Social functioning and school/ work functioning were indeed significantly and substantially lower in the group with diabetes distress in this study. More research is needed to further understand the relation between diabetes distress and social participation of young adults with T1DM. Emotional management involves, among other things, stress management, positive thinking and mental well-being. ${ }^{37}$ The largest negative effect of diabetes distress was indeed found for emotional functioning. More diabetes distress, therefore, could impair one's self-management skills.

The question is how to support young adults with T1DM who have diabetes distress. Apart from more insight into their sources of stress and support needs, studies researching distress in adults can provide recommendations that might be valid for young adults as well. First, as mentioned above, it is essential to regularly screen young adults for diabetes distress and have attention for and discuss any worries. Second, it is important to consider what healthcare professionals can do to counteract diabetes distress in young adults with T1DM. ${ }^{13} \mathrm{~A}$ recent systematic review suggested that experiencing good communication and positive experiences with healthcare professionals is found to be associated with reduced diabetes distress and better self-management outcomes. ${ }^{38}$ Previous studies in young people with chronic conditions have linked positive relationships with and trust in healthcare professionals with more positive outcomes as well. ${ }^{39} 40$ Fisher and colleagues emphasize specific communication styles that healthcare professionals can apply to reduce diabetes distress. ${ }^{38}$ These recommendations are valid for both pediatric and adult care professionals. Transitional care based on good collaboration between pediatric and adult care and harboring a warm reception in adult care could contribute to alleviate the burden for young adults. ${ }^{20}$

\section{Strengths and limitations}

This study addressed a gap in current literature and provided unique insights into diabetes distress around transfer in care. Another strength is the nationwide representation of young adults with T1DM. A possible limitation is that respondents were more often women; some studies suggest there is a significant association between higher diabetes distress and female gender in emerging adults, but the relationship between diabetes distress and gender is not clear. ${ }^{6}$ Therefore, we cannot tell if and how the underrepresentation of males in our study may have influenced our results. Another limitation is that the cross-sectional study design did not allow for exploration of causality; longitudinal studies are needed to identify determinants of diabetes distress in young adults with T1DM. Also, while the response rate in this study was rather low, it appears to be comparable to similar (post transition) studies in young people with T1DM. ${ }^{41}$ Finally, this study did not include any information about diabetes control and the impact on transfer, because the survey and available medical record data were collected in different time periods. Our evaluation study ${ }^{19}$ revealed that mean HbAlc scores did not change over time $(p=0.836)$; they were elevated across the whole study period with no significant differences between pediatric and adult care. It seems relevant to study the association between diabetes control and diabetes distress in future, longitudinal studies.

\section{CONCLUSION}

In this study, more than one-third of the young adults with T1DM experience diabetes distress after transfer to adult care. Differences in diabetes distress are substantial and so are the consequences of having diabetes distress. Those with diabetes distress had less positive transfer experiences, suggesting that the transfer in care could be a source of diabetes distress in young adults. Specific attention is required for reception in adult care, alliance between pediatric and adult care and readiness to transfer. Young adults with diabetes distress also reported less self-management skills and lower HRQoL compared with those without diabetes distress. Structured screening of diabetes distress and attention for and addressing young adults' worries is recommended in both pediatric and adult care.

Acknowledgements We gratefully acknowledge the contributions of the professionals from all participating diabetes teams in facilitating the data collection, and also thank all participating young adults. Maartje van der Slikke, Evelien de Kruif-Hoek, Valérie Wester, Houda Alla and Saskia Hartog are thanked for their assistance during data collection. Ko Hagoort is acknowledged for editorial support.

Contributors JS and AvS conceived the study. All authors participated in its design and execution. JS carried out the literature study and data analysis and drafted the manuscript, while the others contributed to refinement. All authors have read and approved the final version. JS is responsible for the overall content as guarantor.

Funding This study was funded by FNO [101.325]; the Dutch Diabetes Research Foundation [2015.30.1852]. Funding sources had no roles in this study.

Competing interests None declared.

Patient consent for publication Not applicable.

Ethics approval This study involves human participants and was approved by approved by the Medical Ethics Review Board of Erasmus MC with reference number MEC-2014-246. Participants gave informed consent to participate in the study before taking part.

Provenance and peer review Not commissioned; externally peer reviewed.

Data availability statement All data relevant to the study are included in the article.

Open access This is an open access article distributed in accordance with the Creative Commons Attribution Non Commercial (CC BY-NC 4.0) license, which permits others to distribute, remix, adapt, build upon this work non-commercially, and license their derivative works on different terms, provided the original work is properly cited, appropriate credit is given, any changes made indicated, and the use is non-commercial. See: http://creativecommons.org/licenses/by-nc/4.0/. 
ORCID ID

Jane Sattoe http://orcid.org/0000-0002-0433-5022

\section{REFERENCES}

1 lyengar J, Thomas IH, Soleimanpour SA. Transition from pediatric to adult care in emerging adults with type 1 diabetes: a blueprint for effective receivership. Clin Diabetes Endocrinol 2019;5:3.

2 Babler E, Strickland CJ. Moving the journey towards independence: adolescents transitioning to successful diabetes self-management. $J$ Pediatr Nurs 2015;30:648-60.

3 Monaghan M, Helgeson V, Wiebe D. Type 1 diabetes in young adulthood. Curr Diabetes Rev 2015;11:239-50.

4 Weissberg-Benchell J, Wolpert $\mathrm{H}$, Anderson BJ. Transitioning from pediatric to adult care: a new approach to the post-adolescent young person with type 1 diabetes. Diabetes Care 2007;30:2441-6.

5 Vallis M, Willaing I, Holt RIG. Emerging adulthood and type 1 diabetes: insights from the DAWN2 study. Diabet Med 2018;35:203-13.

6 Wentzell K, Vessey JA, Laffel LMB. How do the challenges of emerging adulthood inform our understanding of diabetes distress? an integrative review. Curr Diab Rep 2020;20:1-14.

7 de Wit M, Snoek FJ. Depressive symptoms and unmet psychological needs of Dutch youth with type 1 diabetes: results of a web-survey. Pediatr Diabetes 2011;12:172-6.

8 Buchberger B, Huppertz H, Krabbe L, et al. Symptoms of depression and anxiety in youth with type 1 diabetes: a systematic review and meta-analysis. Psychoneuroendocrinology 2016;70:70-84.

9 Delamater AM. Psychological care of children and adolescents with diabetes. Pediatr Diabetes 2009;10 Suppl 12:175-84.

10 Young V, Eiser C, Johnson B, et al. Eating problems in adolescents with Type 1 diabetes: a systematic review with meta-analysis. Diabet Med 2013;30:189-98.

11 Balfe M, Doyle F, Smith D, et al. What's distressing about having type 1 diabetes? A qualitative study of young adults' perspectives. BMC Endocr Disord 2013;13:25.

12 Delamater AM, de Wit M, McDarby V, et al. ISPAD clinical practice consensus guidelines 2018: psychological care of children and adolescents with type 1 diabetes. Pediatr Diabetes 2018;19 Suppl 27:237-49.

13 Skinner TC, Joensen L, Parkin T. Twenty-Five years of diabetes distress research. Diabet Med 2020;37:393-400.

14 Esbitt SA, Tanenbaum ML, Gonzalez JS. Disentangling clinical depression from diabetes-specific distress: Making sense of the mess we've made. In: Lloyd CE, Pouwer F, Hermanns N, eds. Screening for depression and other psychological problems in diabetes: a practical guide. New York, USA: Springer Publishing, 2013: 27-46

15 Stahl-Pehe A, Glaubitz L, Bächle C, et al. Diabetes distress in young adults with early-onset Type 1 diabetes and its prospective relationship with $\mathrm{HbA}_{1 \mathrm{c}}$ and health status. Diabet Med 2019;36:836-46.

16 Tanenbaum ML, Hanes SJ, Miller KM, et al. Diabetes device use in adults with type 1 diabetes: barriers to uptake and potential intervention targets. Diabetes Care 2017;40:181-7.

17 Downie GA, Mullan BA, Boyes ME, et al. The effect of psychological distress on self-care intention and behaviour in young adults with type 1 diabetes. J Health Psychol 2021;26:543-55.

18 Garvey KC, Foster NC, Agarwal S, et al. Health care transition preparation and experiences in a U.S. national sample of young adults with type 1 diabetes. Diabetes Care 2017;40:317-24.

19 Peeters MAC, Sattoe JNT, Bronner MB, et al. The added value of transition programs in Dutch diabetes care: a controlled evaluation study. J Pediatr Nurs. In Press 2021:S0882-5963(21)00237-2.

20 van Staa A, Peeters M, Sattoe JNT. On your own feet: a practical framework for improving transitional care and young people's self-management. In: Betz C, Coyne I, eds. Switzerland: Transition from Pediatric to Adult Healthcare Services for Adolescents and Young Adults with Long-term ConditionsSpringer, Cham, 2020: 191-228.

21 Sattoe JNT, Peeters MAC, Hilberink SR, et al. Evaluating outpatient transition clinics: a mixed-methods study protocol. BMJ Open 2016;6:e011926.

22 McGuire BE, Morrison TG, Hermanns N, et al. Short-form measures of diabetes-related emotional distress: the Problem Areas in Diabetes Scale (PAID)-5 and PAID-1. Diabetologia 2010;53:66-9.

23 Snoek FJ, Pouwer F, Welch GW, et al. Diabetes-Related emotional distress in Dutch and U.S. diabetic patients: cross-cultural validity of the problem areas in diabetes scale. Diabetes Care 2000;23:1305-9.

24 van Staa A, Sattoe JNT. Young adults' experiences and satisfaction with the transfer of care. J Adolesc Health 2014;55:796-803.

25 Petkov J, Harvey P, Battersby M. The internal consistency and construct validity of the partners in health scale: validation of a patient rated chronic condition self-management measure. Qual Life Res 2010;19:1079-85.

26 Varni JW, Limbers CA. The PedsQL 4.0 generic core scales young adult version: feasibility, reliability and validity in a university student population. J Health Psychol 2009;14:611-22.

27 Lašaite L, Dobrovolskienė R, Danytė E, et al. Diabetes distress in males and females with type 1 diabetes in adolescence and emerging adulthood. J Diabetes Complications 2016;30:1500-5.

28 Hislop AL, Fegan PG, Schlaeppi MJ, et al. Prevalence and associations of psychological distress in young adults with type 1 diabetes. Diabet Med 2008;25:91-6.

29 Fisher L, Polonsky WH, Hessler DM, et al. Understanding the sources of diabetes distress in adults with type 1 diabetes. $J$ Diabetes Complications 2015;29:572-7.

30 Shaw KL, Heath G, Farre A. Transition to Adulthood - Shifting roles between young people with chronic conditions and their parents. In: Self-Management of young people with chronic conditions. Springer, Cham, 2021: 107-34.

31 Sturt J, Dennick K, Due-Christensen M, et al. The detection and management of diabetes distress in people with type 1 diabetes. Curr Diab Rep 2015;15:101.

32 Bronner MB, Peeters MAC, Sattoe JNT, et al. The impact of type 1 diabetes on young adults' health-related quality of life. Health Qual Life Outcomes 2020;18:1-7.

33 Barry-Menkhaus SA, Wagner DV, Riley AR. Small interventions for big change: brief strategies for distress and self-management amongst youth with type 1 diabetes. Curr Diab Rep 2020;20:3

34 Wong SKW, Smith HE, Chua JJS, et al. Effectiveness of selfmanagement interventions in young adults with type 1 and 2 diabetes: a systematic review and meta-analysis. Diabet Med 2020;37:229-41.

35 Lorig KR, Holman H. Self-Management education: history, definition, outcomes, and mechanisms. Ann Behav Med 2003;26:1-7.

36 Sattoe JNT, Hilberink SR, van Staa A, et al. Lagging behind or not? four distinctive social participation patterns among young adults with chronic conditions. J Adolesc Health 2014;54:397-403.

37 Sattoe JNT, Bal MI, Roelofs PDDM, et al. Self-Management interventions for young people with chronic conditions: a systematic overview. Patient Educ Couns 2015;98:704-15.

38 Peimani M, Nasli-Esfahani E, Sadeghi R. Patients' perceptions of patient-provider communication and diabetes care: a systematic review of quantitative and qualitative studies. Chronic IIIn 2020;16:3-22.

39 Monaghan M, Hilliard M, Sweenie R, et al. Transition readiness in adolescents and emerging adults with diabetes: the role of patientprovider communication. Curr Diab Rep 2013;13:900-8.

40 Fisher L, Polonsky WH, Hessler D. Addressing diabetes distress in clinical care: a practical guide. Diabet Med 2019;36:803-12.

41 Garvey KC, Finkelstein JA, Laffel LM, et al. Transition experiences and health care utilization among young adults with type 1 diabetes. Patient Prefer Adherence 2013;7:761. 